\title{
Will BIM's level of development help leave drawings behind?
}

\author{
F. C. Gibson ${ }^{1,2}$ \\ ${ }^{1}$ Virtual Project Delivery, San Francisco, USA \\ ${ }^{2}$ Bechtel Infrastructure, USA
}

\begin{abstract}
Level of Development specification (LOD) has evolved from a relatively obscure technique for cost estimating to an enabling methodology for countless interoperable BIM use cases. With the advent of databases and automation to both capture LOD requirements based on particular use cases and automatic validation of LOD compliance prior to performing use cases, the full power of object based LOD is becoming apparent. Within this new LOD framework, design progress reporting, quality metrics reporting, and design gate control is possible at a BIM object level. With the growing value to customers of BIM models as complete life cycle tools, and the increasing use of digital devices during construction, such as tablets, to view building information, could the end of the drawing sheet paradigm be at hand?
\end{abstract}

Keywords: BIM, Level of Development, $L O D$.

\section{Introduction}

As BIM practice evolves and we find ourselves focusing on topics that may seem esoteric, but in fact become fundamental paradigm shifts in the way we work, it's very helpful to step back and think about the bigger picture. The idea of a "use case" is a common way in the software industry to think about what the user needs to accomplish. In the construction industry, "use case" is a very foreign idea that prevents either understanding or acceptance from many participants.

At Bechtel, we've tried to make understanding and using the concept "use case" more accessible to our colleagues by instead using the idea "Job to be Done" coined by Clayton M. Christensen in his book on innovation [1]. The idea is that in focusing on the job that needs to be done (Job), we can focus on the real 
value we want to create for our customers rather than be distracted by particular tools or solutions that might already be available in the industry. This focus allows us to objectively evaluate tools based on the requirements of the Job, the value we want to add, and encourages the creation or discovery of new tools if none yet exist or are satisfactory.

The question then becomes, what Job does Level of Development (LOD) do for us? How is LOD adding value?

Traditionally in design and engineering, understanding how complete a design was or how good the quality might be was mainly an art, not a science. How did one know if the large stack of sheets was $60 \%$ complete, or $50 \%$ complete or not? How did one know whether the lines drawn were carefully considered and coordinated with other engineering disciplines or not? How much would it cost? Getting an answer to any of these questions required a great deal of time, sheet turning, and educated guessing.

A new production approach entered the scene just over a decade ago Building Information Modelling (BIM). This new tool made generating those stacks of sheets easier and better coordinated through BIM automation. Instead of using lines, BIM used objects that were able to interact and behave accordingly with other objects in modelling the built environment. Could we use this new tool to better understand the cost and reduce some of the guessing? Generating quantities of objects could now happen with the press of a button. Those quantities could then be input into other systems to generate costs - a simple exercise in automated multiplication. But were these costs reliable?

\subsection{LOD evolution}

In addition to the fact that all objects that need to be quantified for costs are not necessarily modelled, objects were also at varying degrees of quality and completeness. One object might be thoroughly coordinated and sized correctly with the correct material applied, while another may be a very rough placeholder object without a defined material or the proper size. As with any computer process, garbage in gave garbage out, and the promise of reliable automatic cost estimates from BIM seemed to fade.

Unwilling to give up so easily, Vico Software, author of a BIM cost estimating product, pushed ahead and developed a new approach to understanding how definitive objects need to be for generating costs with their "Level of Detail" standard using a simple number scale. An object with an LOD of 100 is not very definitive and thus would use a basic area or volume rate. An LOD 200 object would have the right number of items so each could be roughly itemized. At LOD 300, the right number and exact properties of the object allow for precise costing, and at LOD 400, fabrication information is available for the object, and at LOD 500, objects have been installed and field verified to allow payment control systems.

With models using parameters to assign these LOD values accordingly, estimating software could now give accurate costs with tolerances based on the LOD of the objects quantified. But there are several estimates that occur during 


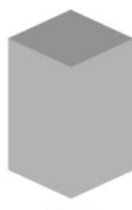

100

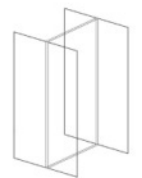

200

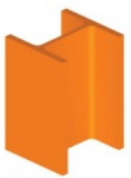

300

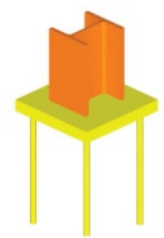

350

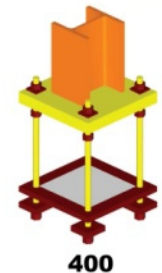

400

Figure 1: BIMForum LOD examples of a steel column.

the evolution of a project growing increasingly precise as the project progresses. How can this evolution be captured? Vico with Webcor Builders created the Model Progression specification which was then refined by the technology subcommittee of the American Institute of Architects (AIA) California Council's Integrated Project Delivery Task Force and adopted by the AIA in 2008 in the form of the E202 document.

During this standardizing process, the AIA set Level of Development as the property to measure as opposed to Level of Detail as the development of the object concerns how definitive the information represented by a BIM object is as opposed to just the amount of information implied by the Level of Detail. In moving to thinking about the reliability of information in objects, the new Level of Development standard opened the door to use the BIM model for many new Jobs, from energy analysis to facility management.

\subsection{LOD specification}

The Level of Development standard has been further refined by BIMForum (the BIMForum's mission is to facilitate and accelerate the adoption of building information modelling (BIM) in the AEC industry. https://bimforum.org/) with their LOD specification [2], "The Level of Development (LOD) specification is a reference that enables practitioners in the AEC Industry to specify and articulate with a high level of clarity the content and reliability of Building Information Models (BIMs) at various stages in the design and construction process."

This comprehensive specification defines the minimum LOD element geometry requirements for a wide variety of objects classified using the UniFormat system. (UniFormat is a standard for classifying building specifications, cost estimating, and cost analysis in the U.S. and Canada managed by the Construction Specification Institute and the Construction Specifications Canada. http://www.csinet.org/uniformat). Additionally, the specification offers the "Model Element Table" Excel spreadsheet to document LOD required for seven different milestones on a given project: SD (Schematic Design), DD (Design Development), CD (Construction Documents), First Estimate, Bid Package Estimate, LEED check, and LEED submission. As part of that Excel framework, additional worksheets are provided to capture and customize attributes required for different object types at different LOD levels. 


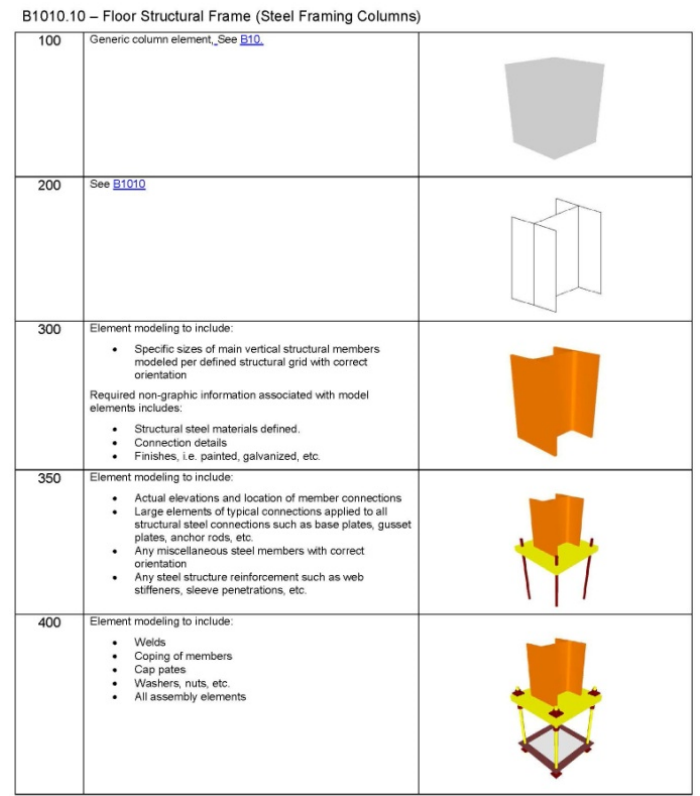

Figure 2: BIMForum floor structural frame LOD specification.

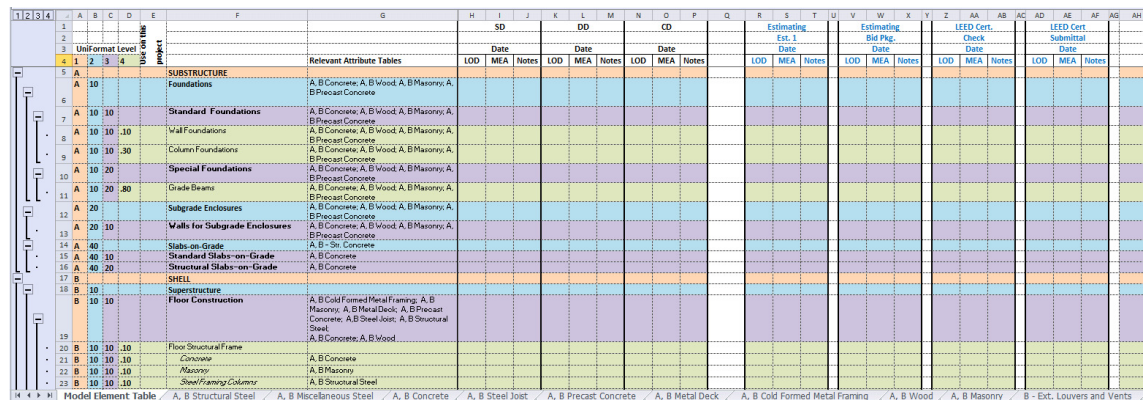

Figure 3: Model element table Excel spreadsheet.

\section{LOD required depends on the Job to be done}

In determining what the required LOD is, the problem with trying to define LOD for a milestone is that a given milestone may need to satisfy several Jobs. For example, when $\mathrm{SD}$ is complete, the preliminary cost estimate and 4D constructability review could also be due. Thus, when defining the LOD required for that milestone, one would have to consider all relevant Jobs that apply and then pick the highest LOD requirement. Any changes made to the milestone later, such as adding or removing Jobs that apply, would require manually going through the whole process again. 
When thinking about LOD requirements for a project for a given milestone, the key is to focus on the Jobs needed at that milestone. For each Job needed, the LOD required by object type will vary. For example, for baseline BIM 3D, the building blocks upon which other 4D, 5D and additional Jobs will depend, the Bechtel Infrastructure Virtual Project Delivery group determined that 14 Jobs are needed, from BIM Authoring to a BIM Content Library to the Engineering Progress Performance Dashboard. Bechtel has established a crossfunctional, multi-discipline scrum team to determine minimum LOD required by Job to be done for each object type.

Because each of these Jobs has different LOD requirements and could potentially occur at different milestones on different projects, the level of complexity in managing what LOD required should be becomes very high.

\subsection{Given the complexity, why bother with LOD?}

As mentioned earlier, without a way to validate the quality of the objects in BIM models, we have no way to know if Jobs such as cross-discipline coordination or automated cost estimates are accurate or helpful. However, the major value of the LOD system is in enabling a move away from the centuries old paper documentation of design to the new paradigm: BIM models viewed and interacted with by any number of devices. Enormous efficiencies in productivity and quality are made possible with the move away from drawings to BIM execution and deliverables.

One thing paper documentation has done very well over the years, however, is to control design change. When a set of documents is issued, for example for construction, the documents include who the architects and engineers of record are, their signatures showing they approve of the documentation, and a date of approval making clear precisely what is approved and at what time. Changes to that design can happen, but only with additional drawings that clearly show what has changed with respect to the last officially issued documents, usually with clouding and revision numbers, and again signed and dated by the designers of record. However, what drawings don't do well is to let the user know whether they are building from the latest set or not.

\subsection{LOD design change control}

Which raises the question: how can design control be maintained with BIM? In the new BIM object paradigm, rather than signing off a sheet in a set of drawings, the designers of record need to sign off on the LOD of the object itself. That object may show up in dozens of views documenting different aspects and detail levels of the installation, used to plan precise procurement schedules, used to calculate exact prices, used to precisely simulate construction sequences, and so on. This object, like the paper documents before it, once validated by the designers of record, effectively becomes the agreed to scope of work with all the force and implications of the contract.

To accomplish this control, the BIM authoring tool must have the ability to authenticate approval by the designer of record as well as lock the state of that 
object including the LOD value. During the design evolution for example, that object will be changed as it moves from the authenticated 200 LOD value to the more developed but not yet authenticated 300 LOD value. Once any change is made to an authenticated object, the BIM authoring tool must capture that change of state and present that object and others to the designer of record for sign off at the higher LOD level.

Additionally, the BIM authoring tool must be able to completely lock objects from any editing at key points such as when an object is released for construction. At that point, a formalized process in the BIM authoring tool of unlocking the object for further changes would only be possible with designer of record approval given the implications of the impact of changes to the model once construction has started. In all cases, the official record of the object's authenticated approval would be embedded in the parameters of the object. Ideally, the BIM authoring tool would capture the complete history of the evolution of the object in the system such that a user could go to a certain point in time and observe what the state of the model was at that point. However, if this is not easily done, simply archiving the model at key milestone dates will serve the same purpose.

\section{LOD system automation}

The value of implementing an LOD system is very high, however, a fully automated approach to both managing and auditing LOD is required to make the approach feasible. The first step is to create a database to capture the Jobs to be done including the associated LOD requirements by object type. The exercise of identifying minimum LOD requirements for each Job is time consuming and represents considerable value once completed.

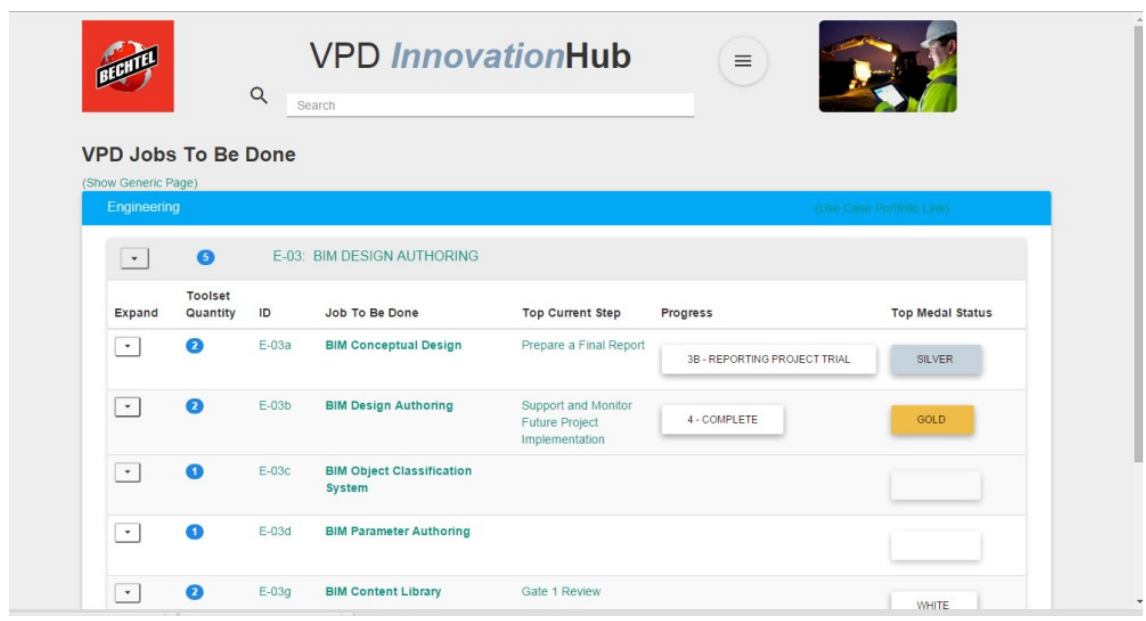

Figure 4: Bechtel's internal InnovationHub graph database. 
The second step is to associate those Jobs with the milestones on a project in the database. The system can then dynamically generate the highest LOD required among all object types for the set of all Jobs required at each milestone. Because the LOD required are specified using the UniFormat classification system, the objects in the model require either UniFormat parameter values for each object or a compatible classification system such as OmniClass table 21. (The OmniClass Construction Classification System (known as OmniClass ${ }^{\mathrm{TM}}$ or OCCS) is a classification system for the construction industry: http://omniclass.org/). Ideally these classifications are built into the BIM content library. The LOD required values can then be mapped into every object in the BIM model's LOD required parameter with an automated parameter authoring system such as Assemble using the classification as the key link. (Assemble Systems cloud based automated parameter authoring/auditing and BIM visualization tool: http://assemblesystems.com/)

\subsection{Visualizing and auditing LOD}

Once LOD requirements are captured, real-time views of the BIM model can be created by automatically color-coding objects based on their current LOD relative to the required LOD. Additionally, real-time schedules can report on satisfying all LOD requirements as a percentage of material volume modelled or total quantity of objects modelled.

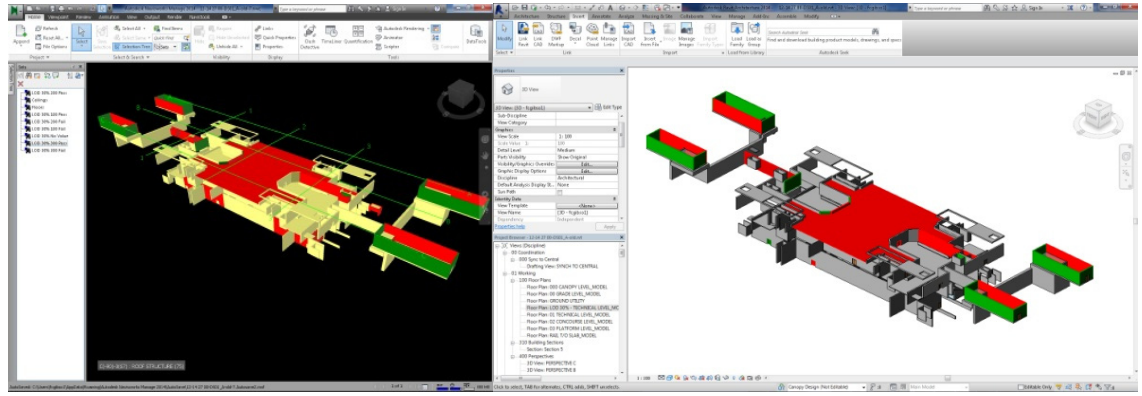

Figure 5: Real-time dynamic LOD color-coded views (Navisworks, Revit).

Table 1: Dynamic LOD completeness schedule (Revit).

\begin{tabular}{|c|c|c|c|c|}
\hline \multicolumn{4}{|c|}{$30 \%$ Milestone Engineering Complete Percentage } \\
\hline $\begin{array}{c}\text { Material: } \\
\text { Volume }\end{array}$ & Quantity & Pass & $\begin{array}{c}\text { By Material \% } \\
\text { Complete }\end{array}$ & $\begin{array}{c}\text { By Quantity \% } \\
\text { Complete }\end{array}$ \\
\hline
\end{tabular}

\begin{tabular}{|l|l|l|l|l|}
\hline $3963.96 \mathrm{~m}^{3}$ & 480 & & $61 \%$ & $92 \%$ \\
\hline $604.01 \mathrm{~m}^{3}$ & 8 & No & $9 \%$ & $2 \%$ \\
\hline $1954.19 \mathrm{~m}^{3}$ & 35 & Yes & $30 \%$ & $7 \%$ \\
\hline $6522.16 \mathrm{~m}^{3}$ & 523 & &
\end{tabular}


When combining this data with estimated total quantities for the facility and comparing with LOD requirements met, meaningful engineering progress reporting can be accomplished. To enable this automated analysis of LOD in the model, a minimum of two parameters must be populated: Current LOD and Required LOD.

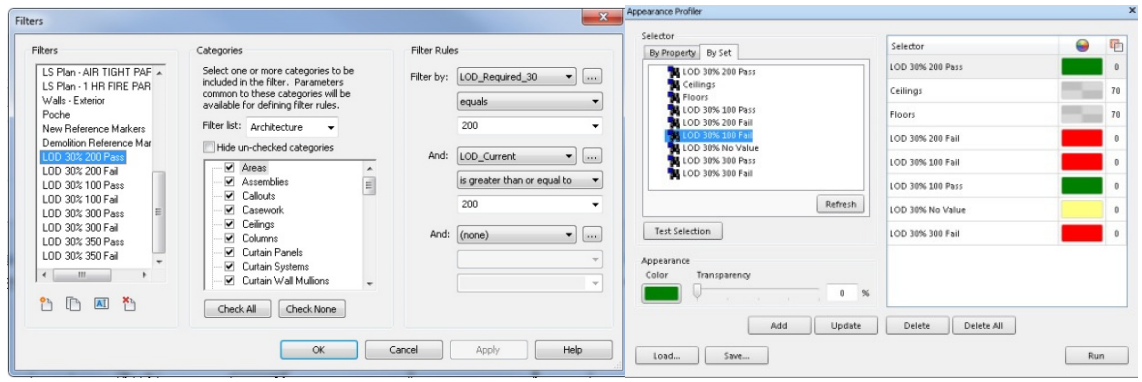

Figure 6: Setting color-coding Logic (Revit, Navisworks).

\section{Project management integration}

For many in project management, the very technical BIM effort can seem impervious to clear understanding by non-technical specialists, and this lack of visibility of the process can lead to real project execution risks. A key to the success of both the BIM and LOD approach is to integrate its use into the standard execution and management of the project.

Some organizations use a structured project review/evaluation program with "gates" that each must be passed through during the execution of the project to proceed to the next gate. At Bechtel, eight key gates have been identified including minimum requirements for each. The key Jobs that apply at each gate with their LOD requirements, once added to the Gate procedure, become a minimum requirement for all projects. This requirement then alerts Project Management to the importance of managing these Jobs like all other activities and deliverables.

Using metrics automatically harvested from the BIM effort such as LOD compliance levels, parameter quality, clashes, percentage of modelling complete, and others, dashboards can be created that allow clear visibility into the progress and quality of the project without the need for any BIM technical skills. With this clear visibility, not only is execution risk removed from managing in the BIM environment, but project management achieves a much clearer and more immediate view of the current state of the project not possible with legacy paper methods.

\section{The future of LOD}

What if we could bring together the entire project team right after conceptual design, from architects and engineers to contractors, trades, and the owner, to 
collaboratively work out how to deliver the project in the leanest way possible? The idea is to eliminate significant rework by only modelling once rather than continually changing the model throughout the design process. The key reason for modelling changes is that information was missing or inadequate to model correctly the first time. Clive Jordan at the Irvine Company has pushed this idea into a multi-stage delivery process (roughly 18 stages) where the collaborative team at the beginning determines the exact information dependencies between stages [3]. Thus at each stage, all the information needed is available and dependent modelling is complete, so that the modelling required for that stage can be completed to fabrication level if required. Why have the mechanical engineer create a design intent model, and then the mechanical trade produce a fabrication model when instead we can just create the fabrication model once? If we combine this idea with a focus on the job to be done and the dependencies between jobs, we can establish a scalable approach to enabling a lean "model it once" paradigm after concept design is complete. The result is a dramatic reduction in waste and increase in design and construction quality with an accompanying reduction in cost and reduction in time needed to complete the project.

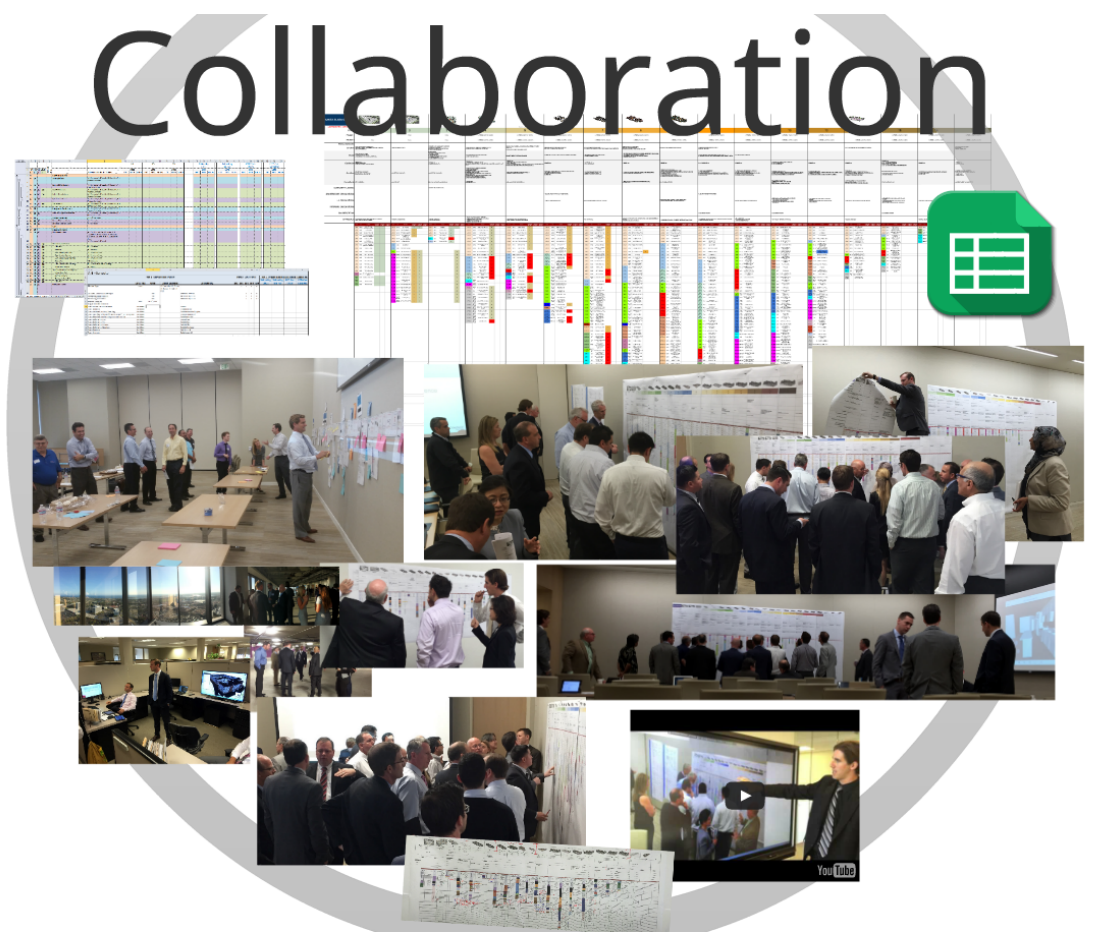

Figure 7: Collaborative pull-planning [3]. 


\section{Summary}

With the growing use of tablets on site to display building information and the growing use of BIM techniques to control the content and quality of projects, one might ask if the need for drawings is rapidly declining? The transition from physical paper to digital drawings has long been underway with the growing use of pdf document files and other digital 2D document formats over the past few years. Generating these digital documents from the BIM environment requires setting up virtual sheets that capture various views for output. This drawing sheet paradigm is still a staple of most engineering production and quality and content control. The drawings are the deliverables and contract documents.

With the recent and increasing growth of BIM Jobs to be done, customers are valuing the BIM model more and more as a facility lifecycle tool rather than something discarded after construction is complete. The BIM model is already becoming a key deliverable.

Once tablets become ubiquitous during construction and LOD systems are used as part of the standard engineering workflow, the drawing sheet paradigm is no longer useful. Perhaps the new deliverable will be geographically located and visualized model information displaying exactly what is needed for the user with instructions on how to achieve the next state of construction rather than simply showing the final intended result. Finally, a dynamic merging of engineering, procurement and construction information with the schedule of the project that transitions seamlessly to operations. Beyond the clear and immediate productivity and quality benefits, it is hard to predict what new possibilities will open up as we finally transition away from legacy paper to the new and expanding paradigm of BIM using LOD.

\section{References}

[1] Christensen, Clayton M., The Innovators Solution: Creating and Sustaining Successful Growth, Harvard Business School Publishing Corporation, Chapter 3, 2003.

[2] BIMForum Level of Development (LOD) specification. https://bimforum.org/lod/

[3] "How to Optimize The Design-Construct-Operate Workflow for the Good of the Entire Project Lifecycle", BIM Integration Congress 2015, San Francisco, Clive Jordan, Irvine Company. 